\title{
Bioinformatics analysis of abnormal DNA methylation in muscle samples from monozygotic twins discordant for type $\mathbf{2}$ diabetes
}

\author{
FEI LIU ${ }^{1}$, QIANQIAN SUN ${ }^{2}$, LINGXIAO WANG ${ }^{3}$, SHUANGSHUANG NIE $^{4}$ and JUN LI ${ }^{2}$ \\ Departments of ${ }^{1}$ Nephrology and ${ }^{2}$ Geriatrics, West China Hospital of Sichuan University; \\ ${ }^{3}$ Geriatric Department, The Fifth People's Hospital of Chengdu City; ${ }^{4}$ Department of Geriatrics, \\ West China Medical College of Sichuan University, Chengdu, Sichuan 610041, P.R. China
}

Received April 1, 2014; Accepted February 6, 2015

DOI: $10.3892 / \mathrm{mmr} .2015 .3452$

\begin{abstract}
The present study aimed to examine the changes in DNA methylation of gene promoters associated with type 2 diabetes (T2D). The DNA methylation profile dataset GSE38291 was downloaded from the Gene Expression Omnibus database. A paired t-test was used to analyze differences in the DNA methylation of gene promoters between T2D and normal muscle samples. Gene Ontology (GO) enrichment analysis was performed using online tool, The Database for Annotation, Visualization and Integrated Discovery. Whole-Genome rVISTA was used to analyze the enriched transcription factor (TF) binding sites upstream of the transcription start site in the differentially methylated genes. A total of 38 genes, including Sirtuin 1, $\mathrm{N}$-acetyltransferase 6 , phospholipase A2 group XIIB and nuclear factor of activated $\mathrm{T}$ cells calcineurin-dependent 1 , were identified to be differentially methylated between these two groups. One GO term, DNA geometric change (GO:0032392), was significantly enriched $(\mathrm{P}<0.05)$ by the hyper-methylated genes. In addition, the binding sites of one gene, zinc finger E-box binding homeobox 1, and three TFs, methyl CpG binding protein 2, TFEB and TFAP4, were significantly enriched in the hyper- and hypo-methylated genes, respectively. The resulting T2D-associated genes and potential TFs provided a novel insight into the molecular mechanisms underlying the pathology of T2D. These genes may become promising target genes for the development of treatments for $\mathrm{T} 2 \mathrm{D}$.
\end{abstract}

Correspondence to: Dr Jun Li, Department of Geriatrics, West China Hospital of Sichuan University, 37 Guo Xue Xiang, Chengdu, Sichuan 610041, P.R. China

E-mail: junlijlil@163.com

Key words: type 2 diabetes, differentially methylated genes, gene ontology enrichment analysis, transcription factors

\section{Introduction}

In recent decades, the incidence and prevalence of type 2 diabetes (T2D) has significantly increased worldwide, particularly among those aged between 35 and 40 years old (1). Long-term diabetes frequently induces vascular changes and dysfunction, and diabetic vascular complications are a major cause of morbidity and mortality amongst patients with diabetes (2).

Dysfunction of the $\beta$ cells of the Islets of Langerhans and insulin-resistance are the main factors underlying the development of T2D (3). In addition, other factors, including inheritance, ethnicity and age, are all associated with the incidence of T2D (4). A previous study identified that the metabolism of glucose and fatty acids by skeletal muscle is impaired in T2D (5). Reduced glucose transport and phosphorylation, as well as reduced rates of glycogen synthesis are common manifestations of insulin-resistant glucose metabolism (6). However, abnormal fatty acid metabolism involves the increased accumulation of triglycerides and other lipids (7), as well as the dysregulation of lipid oxidation under fasting and insulin-stimulated conditions (8). Consequently, the dysfunction of metabolism in muscles may potentially indicate T2D.

DNA methylation is a key epigenetic modification of the genome, which is involved in numerous cellular processes, including cell differentiation and metabolism $(9,10)$. Consistent with these significant roles, associations between aberrant DNA methylation and a growing number of human diseases have been identified (11). In addition, abnormal methylation of several genes, including cytochrome $c$ oxidase polypeptide 7A1, nicotinamide adenine dinucleotide dehydrogenase (ubiquinone) $1 \beta$ subcomplex subunit 6 , peroxisome proliferator-activated receptor $\gamma$ coactivator (PGC) $1-\alpha$ and PGC-1 $\beta$, have been observed in tissues from the skeletal muscles of individuals with type 2 diabetes (12-15).

The development of methylation chips has provided a more efficient approach for the detection of global changes in DNA methylation. Consequently, the current study utilized microarray data derived from the muscle samples of eleven monozygotic twin pairs, discordant for T2D, to detect the global changes in DNA methylation of gene promoters associated with T2D. Gene Ontology (GO) enrichment analysis 


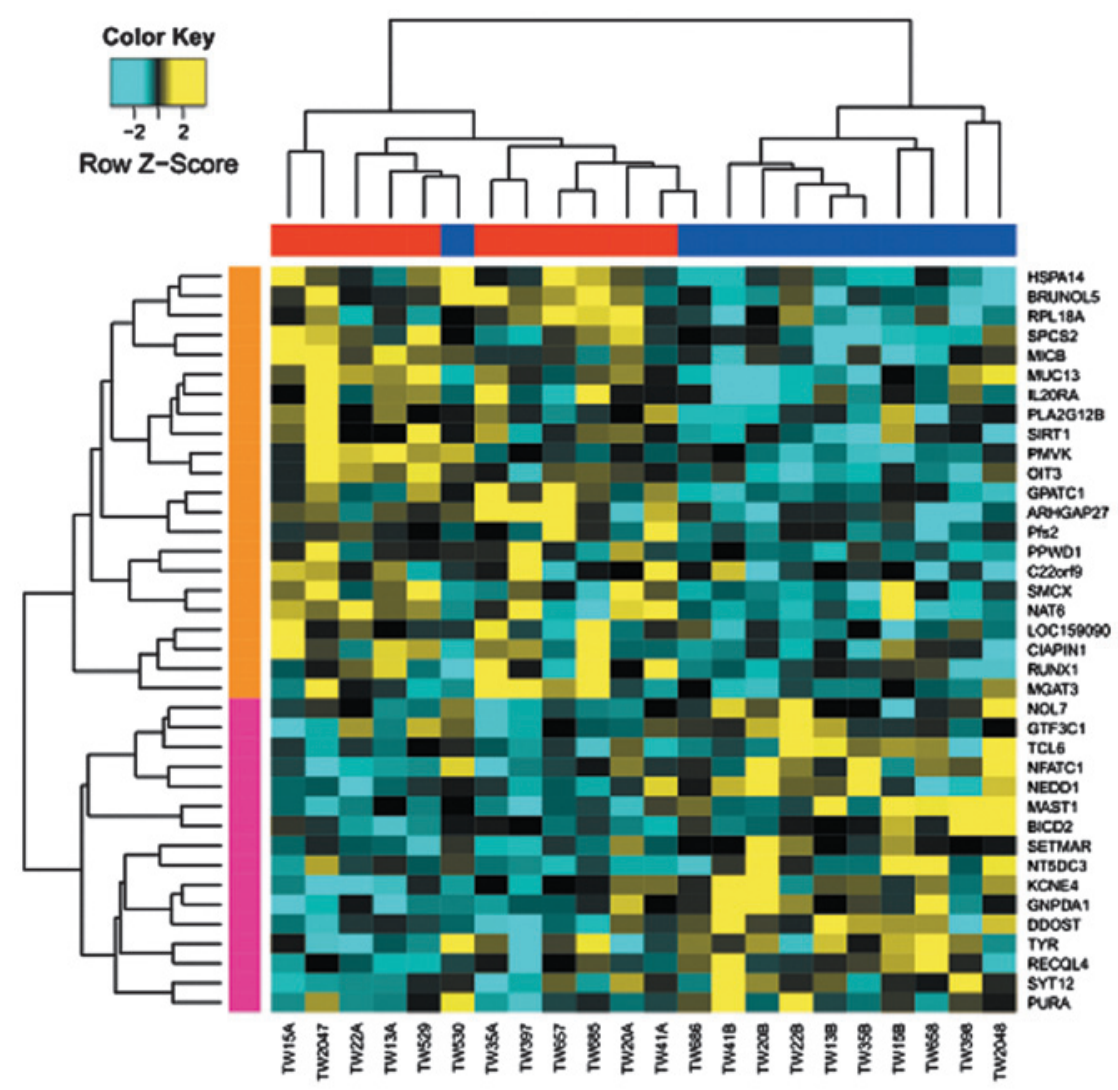

Figure 1. Heat map of differentially methylated genes associated with T2D. Orange and pink columns on the left indicate the hyper- and hypo-methylated genes, respectively, in the T2D group. Red and blue columns on the top indicate the T2D and normal samples, respectively. Yellow and blue boxes indicate the up- and down-regulation of the methylation signal in the T2D group, respectively. T2D, type 2 diabetes.

and prediction analysis of binding sites for potential transcription factors (TFs) were performed in order to investigate the molecular mechanisms involved in T2D.

\section{Materials and methods}

Data acquisition. The DNA methylation dataset, GSE38291 (16), comprising data regarding 11 pairs of monozygotic twins discordant for type 2 diabetes, was downloaded from the Gene Expression Omnibus (http://www. ncbi.nlm.nih.gov/geo/) database. Each pair of monozygotic twins included one individual with T2D and one normal individual (GSM938065-GSM938086). The Illumina HumanMethylation27 BeadChips (Illumina Inc., San Diego, CA, USA), containing a pair of methylated and unmethylated probes designed for each $\mathrm{CpG}$ site, were used to detect the DNA methylation in the gene promoter region.

Quantification of DNA methylation signals. The raw intensities of methylated and unmethylated probes for each sample were extracted based on the Illumina HumanMethylation27 BeadChip. The intensity data were then normalized using quantile normalization. The $\mathrm{M}$-value of each $\mathrm{CpG}$ site was calculated as the $\log _{2}$ ratio of the normalized intensities of methylated probe versus unmethylated probe (17). The M-values of all probes in the promoter of a single gene were calculated and the mid-value was considered to be the methylation level of this gene promoter.
Analysis of differentially methylated genes. A paired samples t-test was employed to analyze the differentially methylated levels of gene promoter between T2D and normal muscle samples. $\mathrm{P}<0.05$ and a fold change of $>1.3$ were considered to indicate the cutoff criteria.

GO enrichment analysis. GO enrichment analysis was performed on the differentially methylated genes for biological processes and cellular components using the online bioinformatics resource: The Database for Annotation, Visualization and Integrated Discovery version 6.7 (http://david.abcc.ncifcrf. gov/) (18). GO terms where $\mathrm{P}<0.1$, were considered to indicate significantly enriched GO terms.

Screening for potential TFs. The Whole-Genome rVISTA tool (19) was used to analyze the enriched TF binding sites in the upstream region (200 bp, $500 \mathrm{bp}$ and $1 \mathrm{~kb}$ ) of transcription start sites (TSS) of the abnormal hyper- and hypo-methylated genes. The TFs with a $\mathrm{P}<0.01$ were considered to be significant. Subsequently, the significant TFs obtained from the three regions were compared, and the most common TFs were considered as the final TFs of the target genes.

\section{Results}

Differentially methylated genes. A total of 38 genes were identified to possess differentially methylated regions between T2D and normal monozygotic twins. Specifically, 22 genes, 
Table I. Significant hyper- and hypo-methylated genes in T2D.

\begin{tabular}{|c|c|c|c|}
\hline Gene & $\log _{2}{ }^{\mathrm{FC}}$ & P-value & $\begin{array}{c}\text { Transcription } \\
\text { factor }\end{array}$ \\
\hline \multicolumn{4}{|c|}{ Hypermethylation in T2D } \\
\hline GPATC1 & 0.770 & 0.006 & No \\
\hline SMCX & 0.610 & 0.021 & No \\
\hline MUC13 & 0.588 & 0.002 & No \\
\hline PMVK & 0.569 & 0.042 & No \\
\hline RPL18A & 0.503 & 0.019 & No \\
\hline IL20RA & 0.486 & 0.025 & No \\
\hline NAT6 & 0.481 & 0.006 & No \\
\hline OIT3 & 0.472 & 0.011 & No \\
\hline ARHGAP27 & 0.469 & 0.009 & No \\
\hline RUNX1 & 0.468 & 0.015 & Yes \\
\hline PFS2 & 0.466 & 0.038 & No \\
\hline BRUNOL5 & 0.462 & 0.005 & No \\
\hline CIAPIN1 & 0.443 & 0.012 & No \\
\hline SPCS2 & 0.436 & 0.016 & No \\
\hline MICB & 0.432 & 0.022 & No \\
\hline MGAT3 & 0.431 & 0.039 & No \\
\hline HSPA14 & 0.430 & 0.024 & No \\
\hline LOC159090 & 0.426 & 0.049 & No \\
\hline SIRT1 & 0.419 & 0.011 & No \\
\hline C22orf9 & 0.413 & 0.039 & No \\
\hline PLA2G12B & 0.409 & 0.029 & No \\
\hline PPWD1 & 0.401 & 0.047 & No \\
\hline \multicolumn{4}{|c|}{ Hypomethylation in T2D } \\
\hline RECQL4 & -0.404 & 0.037 & No \\
\hline SYT12 & -0.405 & 0.030 & No \\
\hline GTF3C1 & -0.405 & 0.043 & No \\
\hline NT5DC3 & -0.406 & 0.039 & No \\
\hline NFATC1 & -0.406 & 0.002 & Yes \\
\hline SETMAR & -0.412 & 0.025 & No \\
\hline NOL7 & -0.421 & 0.031 & No \\
\hline BICD2 & -0.424 & 0.032 & No \\
\hline TCL6 & -0.430 & 0.031 & No \\
\hline KCNE4 & -0.431 & 0.045 & No \\
\hline DDOST & -0.448 & 0.004 & No \\
\hline MAST1 & -0.450 & 0.005 & No \\
\hline TYR & -0.451 & 0.020 & No \\
\hline PURA & -0.468 & 0.038 & No \\
\hline GNPDA1 & -0.471 & 0.022 & No \\
\hline NEDD1 & -0.472 & 0.037 & No \\
\hline
\end{tabular}

T2D, type 2 diabetes.

including SIRT1 (Sirtuin1), NAT6 (N-acetyltransferase 6) and PLA2G12B (phospholipase A2 group XIIB), were hyper-methylated in the T2D group; while 16 genes, including nuclear factor of activated T cells calcineurin-dependent 1 (NFATC1) and microtubule associated serine/threonine kinase 1 , were hypo-methylated (Table I, Fig. 1). In addition, two TFs were identified in the hyper- and hypo- methylated genes, respec- tively, based on the public TF database (Table I) (20). These two TFs were Runt-related transcription factor 1 and NFATC1, which possessed significant hyper- and hypo-methylated regions in $\mathrm{T} 2 \mathrm{D}$ muscles.

GO enrichment analysis. According to the GO enrichment analysis, hyper-methylated genes were enriched in three GO 
Table II. Significantly enriched GO terms of differentially methylated genes in T2D.

\begin{tabular}{llccc}
\hline Methylation status & \multicolumn{1}{c}{ GO term } & $\begin{array}{c}\text { Gene } \\
\text { counts }\end{array}$ & P-value & Genes \\
\hline Hypermethylated genes in T2D & $\begin{array}{l}\text { GO: 0048585-negative regulation of } \\
\text { response to stimulus }\end{array}$ & 2 & 0.072 & MICB, SIRT1 \\
& GO: 0008219-cell death & 3 & 0.096 & MICB, CIAPIN1, SIRT1 \\
& GO: 0016265-death & 3 & 0.097 & MICB, CIAPIN1, SIRT1 \\
Hypomethylated genes in T2D & GO: 0032392-DNA geometric change & 2 & 0.015 & RECQL4, PURA \\
& GO: 0006259-DNA metabolic process & 3 & 0.061 & RECQL4, SETMAR, PURA \\
& GO: 0006310-DNA recombination & 2 & 0.082 & RECQL4, SETMAR
\end{tabular}

T2D, type 2 diabetes; GO, gene ontology.

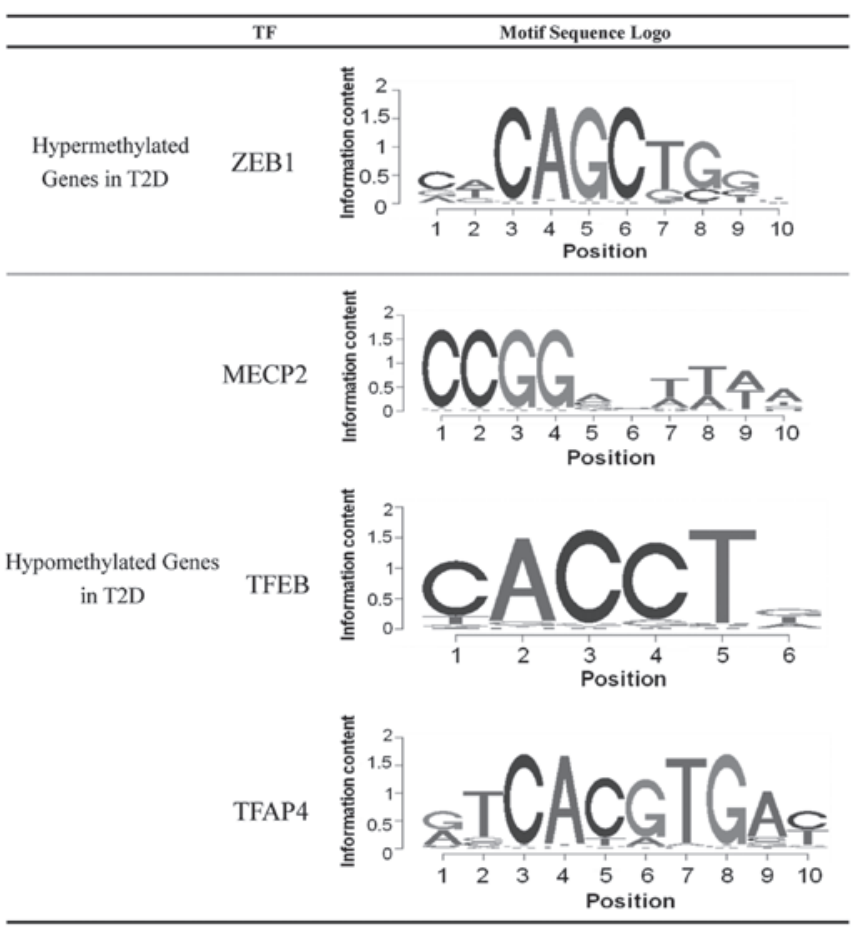

Figure 2. Potential transcription factors for differentially methylated genes. $\mathrm{TF}$, transcription factor; MECP2, methyl CpG binding protein 2; ZEB1, zinc finger E-box binding homeobox 1; T2D, type 2 diabetes.

terms $(\mathrm{P}<0.1$, Table II). The negative regulation of response to stimulus (GO: 0048585), which was associated with a process that stops, prevents or reduces the frequency, rate or extent of a response to a stimulus, was enriched by three genes, which may be significant in T2D pathology. The terms cell death (GO: 0008219) and death (GO: 0016265), refer to the biological processes that result in permanent cessation of all vital functions of a cell. By contrast, the hypo-methylated genes were significantly enriched in three classes of GO terms. DNA geometric change (GO: 0032392) is a term regarding the process in which a transformation is induced in the geometry of a DNA double helix, resulting in a change in twist and/or writhe. There were three genes enriched for DNA metabolic process (GO: 0006259), which is associated with cellular metabolic processes involving deoxyribonucleic acid. Finally, two genes were highly enriched for DNA recombination (GO: 0006310), involving the process in which a new genotype is formed via a reassortment of genes resulting in novel gene combinations.

Screening for potential TFs. The enrichment of TF binding sites in the upstream region of the abnormal hyper- and hypo-methylated genes was analyzed. Multiple TF binding sites were significantly enriched among the differentially methylated genes, and four corresponding TFs were subsequently identified. As shown in Fig. 2, only the TF zinc finger E-box binding homeobox 1 (ZEB1) was identified as possessing a large probability to bind and regulate the hyper-methylated genes. While the binding sites of three TFs, methyl $\mathrm{CpG}$ binding protein 2 (MECP2), TFEB and TFAP4, were enriched in the hypo-methylated genes.

\section{Discussion}

The differences in global DNA methylation in muscle tissues from 11 monozygotic twin pairs discordant for T2D were detected using microarray data. The utilization of monozygotic twins facilitates investigation of the abnormal DNA methylation induced by environmental factors, but not hereditary factors, in T2D patients.

The significantly hyper- and hypo-methylated genes identified in the current study may provide several novel candidate genes associated with the occurrence of T2D. SIRT1, an NAD(+)-dependent histone deacetylase, which was hyper-methylated according to the present results, was observed to regulate glucose/lipid metabolism through its deacetylase activity on numerous substrates $(21,22)$. In addition, SIRT1 positively regulates insulin secretion in pancreatic $\beta$ cells (23), and protects cells from oxidative stress and inflammation. Consequently, the methylation of the SIRT1 gene may be a significant gene modification involved in the occurrence of T2D. The other two genes, NAT6 and PLA2G12B, which were also hyper-methylated in T2D muscle, are involved in the metabolism of lipids (24). PLA2G12B belongs to the PLA2 family, and catalyzes the hydrolysis of glycolipids to release free fatty acids and lysophospholipids (25). NAT6 encodes a cytoplasmic $\mathrm{N}$-acetyltransferase protein, with substrate specificity for proteins with an N-terminal methionine (26). Additionally, these two genes are involved in the 
pathway of glycerophospholipid metabolism according to the Kyoto Encyclopedia of Genes and Genomes pathway analysis (http://www.genome.jp/kegg/). All of this cumulative evidence has suggested that the hyper-methylation of PLA2G12B and NAT6 may result in the dysfunction of glycerophospholipid metabolism leading to hyperlipidemia in T2D patients.

Of the hypo-methylated genes, NFATC1 is particularly notable for its potential association with T2D. A previous study revealed that mice with a $\beta$-cell-specific deletion of the calcineurin b1 (CNB1) subunit developed age-dependent diabetes characterized by decreased $\beta$-cell proliferation and mass, reduced pancreatic insulin content and hypo-insulinemia. The expression of active NFATC1 in CNB1-deficient $\beta$ cells may rescue these defects and prevent diabetes (27). This evidence indicated the positive role of NFATC1 in the pathogenesis or the potential therapeutic role in diabetes.

According to the enrichment of TF binding sites in the upstream region of the differentially methylated genes, it was demonstrated that the transcription factor ZEB1 was significantly enriched for its binding to the hyper-methylated genes identified in the current study. Papadopoulou et al (28) hypothesized that transfection of ZEB1 cDNA may induce a reduction in $\mathrm{B}$ cell lymphoma 6 (BCL6) expression, and that siRNA-mediated knockdown of ZEB1 may lead to an increase in BCL6 mRNA expression (28). BCL-6 represses menin, a product of the Men1 gene, expression. Transgenic menin overexpression in $\beta$ cells prevents islet expansion, which leads to maternal hyperglycemia (29). Therefore, transgenic menin mice exhibit features of human gestational diabetes (29). For these reasons, ZEB1, as a transcription factor, may be involved in the pathogenesis of T2D. The binding site of MECP2 was significantly enriched by the hypo-methylated genes in the present study. MECP2 is capable of binding specifically to methylated DNA to suppress transcription from methylated gene promoters (30). The DNA methylation of genes, as the major modification of eukaryotic genomes (31), was repressed by MECP2, indicating a key function for MECP2 in the regulation of methylated genes. It was therefore hypothesized that MECP2 may be involved in the process of T2D via regulation of the hypo-methylated genes; however, this hypothesis requires further investigation for confirmation. The present results also identified two candidate TFs (TFEB and TFAP4), which may regulate the hypo-methylated genes in T2D. To the best of our knowledge, few studies have investigated the association between these two TFs and T2D, and therefore future studies should focus on elucidating this association.

In conclusion, a bioinformatics analysis of the differentially methylated genes in muscle tissues from 11 monozygotic twin pairs discordant for T2D was performed. The identified diabetes-associated genes (SIRT1, NAT6, PLA2G12B and NFATC1) and potential TFs provided a novel insight into the molecular mechanism underlying the occurrence of T2D. These genes may become promising target genes in the development of novel treatments for T2D.

\section{Acknowledgements}

The present study was supported by a grant from a project supported by the China National Natural Science
Foundation (grant no. 81300260) and the Foundation of Science and Technology Department of Sichuan Province (grant no. 14ZC1146).

\section{References}

1. Parving H-H, Brenner BM, McMurray JJ, et al; ALTITUDE Investigators: Cardiorenal end points in a trial of aliskiren for type 2 diabetes. N Engl J Med 367: 2204-2213, 2012.

2. Kitada M and Koya D: SIRT1 in type 2 diabetes: Mechanisms and therapeutic potential. Diabetes Metab J 37: 315-325, 2013.

3. Kahn SE: The relative contributions of insulin resistance and beta-cell dysfunction to the pathophysiology of Type 2 diabetes. Diabetologia 46: 3-19, 2003.

4. Spranger J, Kroke A, Möhlig M, et al: Adiponectin and protection against type 2 diabetes mellitus. Lancet 361: 226-228, 2003.

5. Hofs $\varnothing \mathrm{D}$, Jenssen T, Hager H, Røislien J and Hjelmesaeth J: Fasting plasma glucose in the screening for type 2 diabetes in morbidly obese subjects. Obes Surg 20: 302-307, 2010.

6. Zhang P, Zhang X, Brown J, et al: Global healthcare expenditure on diabetes for 2010 and 2030. Diabetes Res Clin Pract 87: 293-301, 2010.

7. Liebl A, Mata M and Eschwège E; ODE-2 Advisory Board: Evaluation of risk factors for development of complications in Type II diabetes in Europe. Diabetologia 45: S23-S28, 2002.

8. Sjöström L, Lindroos A-K, Peltonen M, et al; Swedish Obese Subjects Study Scientific Group: Lifestyle, diabetes, and cardiovascular risk factors 10 years after bariatric surgery. N Engl J Med 351: 2683-2693, 2004.

9. Barres R and Zierath JR: DNA methylation in metabolic disorders. Am J Clin Nutr 93: 897S-900S, 2011.

10. Takizawa T, Nakashima K, Namihira M, et al: DNA methylation is a critical cell-intrinsic determinant of astrocyte differentiation in the fetal brain. Dev Cell 1: 749-758, 2001.

11. Robertson KD: DNA methylation and human disease. Nat Rev Genet 6: 597-610, 2005.

12. Rönn T, Poulsen P, Hansson O, et al: Age influences DNA methylation and gene expression of COX7A1 in human skeletal muscle. Diabetologia 51: 1159-1168, 2008.

13. Ling C, Poulsen P, Carlsson E, et al: Multiple environmental and genetic factors influence skeletal muscle PGC- $1 \alpha$ and PGC-1 $\beta$ gene expression in twins. J Clin Invest 114: 1518-1526, 2004.

14. Ling C, Poulsen P, Simonsson S, et al: Genetic and epigenetic factors are associated with expression of respiratory chain component NDUFB6 in human skeletal muscle. J Clin Invest 117: 3427-3435, 2007.

15. Jensen CB, Storgaard H, Madsbad S, Richter EA and Vaag AA: Altered skeletal muscle fiber composition and size precede whole-body insulin resistance in young men with low birth weight. J Clin Endocrinol Metab 92: 1530-1534, 2007.

16. Barrett T, Wilhite SE, Ledoux P, et al: NCBI GEO: archive for functional genomics data sets - update. Nucleic Acids Res 41: D991-D995, 2013.

17. Du P, Zhang X, Huang CC, et al: Comparison of Beta-value and M-value methods for quantifying methylation levels by microarray analysis. BMC Bioinformatics 11: 587, 2010.

18. Da Wei Huang BTS, Sherman BT and Lempicki RA: Systematic and integrative analysis of large gene lists using DAVID bioinformatics resources. Nat Protoc 4: 44-57, 2009.

19. Dubchak I, Munoz M, Poliakov A, et al: Whole-Genome rVISTA: a tool to determine enrichment of transcription factor binding sites in gene promoters from transcriptomic data. Bioinformatics 29: 2059-2061, 2013.

20. Wingender E, Dietze P, Karas H, and Knüppel R: TRANSFAC: A database on transcription factors and their DNA binding sites. Nucleic Acids Res 24: 238-241, 1996.

21. Kume S, Uzu T, Kashiwagi A and Koya D: SIRT1, a calorie restriction mimetic, in a new therapeutic approach for type 2 diabetes mellitus and diabetic vascular complications. Endocr Metab Immune Disord Drug Targets 10: 16-24, 2010.

22. Sharma S, Misra CS, Arumugam S, et al: Antidiabetic activity of resveratrol, a known SIRT1 activator in a genetic model for type 2 diabetes. Phytother Res 25: 67-73, 2011.

23. Bordone L, Motta MC, Picard F, et al: Sirt1 regulates insulin secretion by repressing UCP2 in pancreatic $\beta$ cells. PLoS Biol 4: e31, 2006 
24. Bhakta S, Besra GS, Upton AM, et al: Arylamine $\mathrm{N}$-acetyltransferase is required for synthesis of mycolic acids and complex lipids in Mycobacterium bovis BCG and represents a novel drug target. J Exp Med 199: 1191-1199, 2004.

25. Aljakna A, Choi S, Savage H, et al: Pla2g12b and Hpn are genes identified by mouse ENU mutagenesis that affect HDL cholesterol. PLoS One 7: e43139, 2012.

26. Blum M, Grant DM, Mcbride W, Heim M and Meyer UA: Human arylamine $\mathrm{N}$-acetyltransferase genes: isolation, chromosomal localization, and functional expression. DNA Cell Biol 9: 193-203, 1990

27. Heit JJ, Apelqvist AA, Gu X, et al: Calcineurin/NFAT signalling regulates pancreatic $\beta$-cell growth and function. Nature 443: 345-349, 2006
28. Papadopoulou V, Postigo A, Sánchez-Tilló E, Porter AC and Wagner SD: ZEB1 and CtBP form a repressive complex at a distal promoter element of the BCL6 locus. Biochem J 427: 541-550, 2010.

29. Karnik SK, Chen H, McLean GW, et al: Menin controls growth of pancreatic $\beta$-cells in pregnant mice and promotes gestational diabetes mellitus. Science 318: 806-809, 2007.

30. Nan X, Ng HH, Johnson CA, et al: Transcriptional repression by the methyl-CpG-binding protein $\mathrm{MeCP} 2$ involves a histone deacetylase complex. Nature 393: 386-389, 1998.

31. Li E, Beard C and Jaenisch R: Role for DNA methylation in genomic imprinting. Nature 366: 362-365, 1993. 